\title{
Treatable traits: a step closer to the "holy grail" of asthma control?
}

\author{
William W. Busse \\ Affiliation: Dept of Medicine, Division of Allergy, Pulmonary and Critical Care Medicine, University of \\ Wisconsin School of Medicine and Public Health, Madison, WI, USA.
}

Correspondence: William W. Busse, University of Wisconsin Hospital, K4/910 CSC, MC 9988, 600 Highland Avenue, Madison, WI 53792, USA. E-mail: wwbamedicine.wisc.edu

@ERSpublications

McDonald and colleagues are the first to demonstrate in a clinical trial that treatment of treatable traits in severe asthma improved disease control compared to guideline-directed care http://bit.ly/ 2QXZG6v

Cite this article as: Busse WW. Treatable traits: a step closer to the "holy grail" of asthma control? Eur Respir J 2020; 55: 2000002 [https://doi.org/10.1183/13993003.00002-2020].

Gaining optimal disease control remains a major unmet need for many patients with asthma [1]. Although progress in disease control has occurred with modifications to current guidelines and with newly available biologics, this unmet need is particularly problematic in severe asthma [2].

Landmarks in the journey to more effective asthma treatment approaches began with the development and incorporation of asthma guidelines into clinical practice [3, 4]. Guidelines continue to serve as major directives in defining desirable outcomes of disease control and utilising evidence-based approaches to achieve this goal. The principles of most guideline-based treatment steps, however, represent a "one care approach for all". Consequently, overall outcome achievements often fall short of optimal control [5], despite guideline advances such as those made by the Global Initiative for Asthma in difficult-to-treat and severe asthma [6].

Many reasons account for our shortfalls in achieving an overall well-being for a larger proportion of asthma patients. For example, phenotypic and endotypic features of asthma vary and differentially regulate the pattern and burden of disease for individual patients as well as response to treatment [7]. The concept of asthma as a heterogeneous disease, and the introduction of T2 directed biologics for greater precision of treatment, has provided evidence to strongly suggest that identifying therapeutic targets that are relevant and contribute to pathophysiological characteristics will significantly improve disease control [8]. Recognising that individual patient characteristics are formulated by multiple traits to construct the final asthma profile has therefore spurred efforts to formulate more effective treatment approaches and incorporate paradigms that focus on precise features of disease, whose regulation may translate to greater disease control [9]. This concept is particularly true in severe disease, where a consistent response to current treatments is variable and the burden from uncontrolled illness is the greatest [2].

To improve the efficacy of asthma control while carefully taking into consideration the multifaceted and individualised contributors to the existing disease, AGUSTI et al. [10] published a vanguard and thoughtful perspective: "Precision medicine in airway diseases: moving to clinical practice." Their proposal to identify "treatable traits" as therapeutic targets has become a conceptual promise that more precise and inclusive individualisation of treatment would lead to a greater likelihood of asthma control.

In theory, the identification of multidimensional, treatable traits in asthma also provides an opportunity to dissect the existing complexity of disease in individual patients and identify comorbid traits that contribute 
to the holistic characteristics of existing disease. Based on this concept, an identification of treatable traits would formulate meaningful and personalised therapeutic targets. The application of treatable traits to treatment was, until now, a concept in search of rigorous evaluations to ascertain the efficacy of this approach.

In this issue of the European Respiratory Journal, McDonald et al. [11] have taken a major step toward testing the hypothesis that targeting treatable traits in severe asthma patients represents an effective step forward to gain greater asthma control. From my review and perspective, their current findings, with promises for future implications, are a significant advance in our quest to improve asthma control through personalised treatment approaches. Moreover, their pilot work formulates a model system to implement a multidimensional therapeutic approach that is specific to the multiple and contributing pathophysiological asthma characteristics affecting individual patients.

When I evaluated the findings of McDonald et al. [11], a number of key elements highlighted their proof-of-concept study of treatable-traits directed treatment and suggested to me why their findings represent a significant advance towards precise, comprehensive, and, thus, improved treatment of severe asthma.

Their study design carefully identified and characterised 26 relevant treatable traits, which were grouped into three domains (table 1): pulmonary; extrapulmonary; and risk factors and behavioural traits. From my perspective, this logistic design overview established a comprehensive assessment of the nature and complexity of each individual patient's existing asthma, and provided a "break down" of identified treatable traits into targetable and, presumably, contributing components of their asthma. For each identified treatable trait, specific interventions were proposed (table 3).

Traditionally, and from my personal perspective, the pulmonary category of treatable traits has assumed a principal therapeutic focus as it directs attention, importance and treatment towards accepted key elements of asthma: airway inflammation and airflow obstruction. A prioritisation towards the pulmonary category also arises from a belief that altered pulmonary physiology (airflow obstruction and inflammation) represents a major determinant of clinical manifestations of asthma, including susceptibility for exacerbation [12]. However, the authors' expanded, multidimensional view of treatable traits, including extrapulmonary (e.g. obesity, cardiac history, vocal cord dysfunction) and risk factors and behavioural traits (e.g. smoking, inhaler device, polypharmacy) gives equal and relevant importance to the overall clinical manifestations of asthma. Consequently, these multiple traits should be more appropriately considered as significant contributors to the overall severity of asthma and the belief that "only" by treating each relevant comorbid trait will it be possible to gain optimal disease control. Examples of the traits of the three domains include: pulmonary: airflow limitation and airway inflammation (eosinophilic or neutrophilic); extrapulmonary: systemic inflammation and obesity; and risk factors and behavioural traits: absence of a written plan and smoking.

The investigators recruited 140 participants from their severe asthma clinic; from this population, 28 were randomised to the intervention group and 27 to the control group (table 2). Ongoing care for the control group occurred in a severe asthma clinic which was managed by a collective team of experts in severe asthma and largely followed guideline-directed care. For the intervention group, the investigators identified 26 treatable traits which represented nine pulmonary, nine extrapulmonary, and eight behavioural/risk factors (table 1). There was a mean number of 10.44 treatable traits per participant in the intervention group with the following distribution (mean values): pulmonary (3.01), extrapulmonary (4.85) and behavioural/risk (2.58). The clinical importance and relevance of these selected treatable traits to an overall measure of disease severity was supported by analyses showing that the total number of traits in the three categories correlated significantly with an assessment of health status by the St George Respiratory Questionnaire $(\mathrm{r}=0.61, \mathrm{p}<0.001)$ (figure $4 \mathrm{~b}$ ).

A pivotal logistic member of the intervention team was the nurse case-manager. The case-manager's function appears critical both to the application and success of their interventions and had duties which included a summarisation of the traits identified, coordination of the case discussion with the multidisciplinary team, assessing the importance of each trait from the perspective of both the physician and patient, providing education on the traits and individual treatments, and coordinating the various visits between the multidisciplinary team. The case-manager was truly the conductor of this treatable trait symphony.

The intervention group received treatment for a mean \pm SD of $8.36 \pm 1.92$ traits, which occurred during $5.32 \pm 2.53$ treatment visits over the 16 -week trial; this visit encounter differential compared to $2.37 \pm 2.32$ visits for the control group $(\mathrm{p}<0.001)$. As noted, for each treatable trait, the investigators identified a specific intervention. For example, for the treatable trait of eosinophilic inflammation (sputum eosinophils 
$\geqslant 3 \%$ ), high dose inhaled corticosteroids (ICS) (fluticasone $500 \mu \mathrm{g}$ twice per day) plus systemic corticosteroids (prednisone $12.5 \mathrm{mg}$ per day) were used, with treatment adjusted by re-assessments of sputum cell counts. For neutrophilic inflammation (sputum neutrophils $\geqslant 61 \%$ ), the investigators intervened with azithromycin $250 \mathrm{mg}$ daily for 16 weeks. Finally, systemic inflammation was identified by measures of high-sensitivity C-reactive protein $\left(\geqslant 3 \mathrm{mg} \cdot \mathrm{L}^{-1}\right)$ and treated with rosuvastatin $(20 \mathrm{mg}$ per day for 16 weeks).

The investigators selected as their primary outcome a change in the Asthma Quality of Life Questionnaire (AQLQ) [13] score of $>0.8$ over the 16-week treatment, an endpoint, which, they contend, represents a global measure to assess the benefit of treatment interventions on the overall burden of a patient's asthma. The intervention group had a significant $(\mathrm{p}<0.001)$ mean increase in AQLQ of 0.86 (95\% CI $0.49,1.23)$ compared to $-0.004(95 \%$ CI $-0.29,280$ ) for the control group (figure $5 \mathrm{a}$ ). Moreover, $55.56 \%$ of the intervention group achieved at least a minimally clinically important difference (0.5) in AQLQ versus $22.22 \%(\mathrm{p}=0.02)$ in the control group (figure $5 \mathrm{~b}$ ).

Why do I feel that the findings of McDonald et al. [11] are important and represent an advance for future consideration? As the authors state, and I fully agree, there is a need to rethink treatment approaches for complex diseases like asthma. Their findings [11] lay out guiding steps to achieve an improvement for such outcomes. Second, in addition to being a complex and chronic disease, asthma is multidimensional; focusing on only one or two aspects of contributing factors to a patient's asthma will likely result in less effective treatment outcomes. Consequently, their results support and strengthen the concept of a multidimensional approach. Furthermore, a comprehensive identification of treatable traits for each patient provides both a holistic and a priority treatment approach, which more comprehensively represents what asthma is to an individual patient and how it affects their lifestyle and well-being.

For their approach to succeed, key components and efforts were essential. The inclusion of a care-manager is, as already mentioned and in my opinion, $a$, if not the, key component to coordinate the selected interventions for the identified individual treatable traits. However, the case-manager's efforts represent major time and knowledge commitments over the mean 5.32 visits during the 16 -week trial. There was no quantification of the total time this represented collectively or on an individual basis. What is also not addressed is whether a focus on treatable traits will be practical in clinical medicine, financially feasible, and applicable on a larger scale. However, the study design is a structural guidance to address these and other practical and tactical questions.

Another question is whether this approach could be more tailored to reduce the overall effort but yet capture the desired optimal control. For example, it would be helpful to know whether treatment of particular domains or specific traits provide the greatest benefit and could thus serve as principal priorities: is it pulmonary, extrapulmonary, or behavioural/risk? Current approaches to achieve asthma control in severe disease have largely focused on the pulmonary domain. Data (table 3 ) indicate that one of the most significant areas in which benefit occurred was improved airflow obstruction. An expanding experience with biologics in severe asthma has shown that treatment specifically directed towards components of inflammation is effective in achieving control, including a prevention of exacerbations [14]. Biologics, however, were not an intervention in this study. Given our expanding experiences with current T2-directed biologics, what impact would biologics have on the paradigm of treatable traits? Finally, the study was only 16 weeks and a detection of differences in exacerbations was not a powered outcome, though a favourable trend toward fewer exacerbations in the intervention group was noted (table S7). These limitations and considerations aside, McDonald et al. [11] are to be congratulated as they are the first to systematically explore the effects of targeting treatable traits in severe asthma. We now await larger and longer studies for future directions as we search to gain more complete control of asthma and discover whether the treatable traits approach is a step towards our desired Holy Grail in this respect.

Conflict of interest: W.W. Busse reports personal fees for consultancy from AstraZeneca, GlaxoSmithKline, Novartis, Sanofi and Regeneron, personal fees for data monitoring committee work from Boston Scientific, personal fees for consultancy and data monitoring committee work from Genentech, royalties from Elsevier, outside the submitted work.

\section{References}

1 Price D, Fletcher M, van der Molen T. Asthma control and management in 8,000 European patients: the REcognise Asthma and LInk to Symptoms and Experience (REALISE) survey. NPJ Prim Care Respir Med 2014; 24: 14009 .

2 Chung KF, Wenzel SE, Brozek JL, et al. International ERS/ATS guidelines on definition, evaluation and treatment of severe asthma. Eur Respir J 2014; 43: 343-373.

3 National Asthma Education and Prevention Program. Expert Panel Report 3: Guidelines for the Diagnosis And Management of Asthma. Bethesda, National Institutes of Health, National Heart, Lung and Blood Institute, 2007. Publication no. 07-4051. Available from: www.nhlbi.nih.gov/guidelines/asthma 
4 Global Initiative for Asthma. Global Strategy for Asthma Management and Prevention. 2018. www.ginasthma.org. Date last accessed: 28 March, 2018.

5 Szefler SJ. Asthma across the lifespan: Time for a paradigm shift. J Allergy Clin Immunol 2018; 142: 773-780. Global Initiative for Asthma. Difficult-to-Treat and Severe Asthma. 2019. www.ginasthma.org

7 Ray A, Oriss TB, Wenzel SE. Emerging molecular phenotypes of asthma. Am J Physiol Lung Cell Mol Physiol 2015; 308: L130-L140.

8 Israel E, Reddel HK. Severe and difficult-to-treat asthma in adults. N Engl J Med 2017; 377: 965-976.

9 McDonald VM, Fingleton J, Agusti A, et al. Treatable traits: a new paradigm for 21st century management of chronic airway diseases: Treatable Traits Down Under International Workshop report. Eur Respir J 2019; 53: 1802058.

10 Agusti A, Bafadhel M, Beasley $\mathrm{R}$, et al. Precision medicine in airway diseases: moving to clinical practice. Eur Respir J 2017; 50: 1701655.

11 McDonald VM, Clark VL, Cordova-Rivera L, et al. Targeting treatable traits in severe asthma: a randomised controlled trial. Eur Respir J 2020; 55: 1901509.

12 DiMango E, Rogers L, Reibman J, et al. Risk factors for asthma exacerbation and treatment failure in adults and adolescents with well-controlled asthma during continuation and step-down therapy. Ann Am Thorac Soc 2018; 15: 955-961.

13 Juniper EF, Guyatt GH, Ferrie PJ, et al. Measuring quality of life in asthma. Am Rev Respir Dis 1993; 147: 832-838.

14 Krings JG, McGregor MC, Bacharier LB, et al. Biologics for severe asthma: treatment-specific effects are important in choosing a specific agent. J Allergy Clin Immunol Pract 2019; 7: 1379-1392. 